\title{
Droplet digital PCR as a novel system for the detection of microRNA-34b/c methylation in circulating DNA in malignant pleural mesothelioma
}

\author{
HIROKI SATO ${ }^{1}$, JUNICHI SOH ${ }^{1 *}$, KEISUKE AOE ${ }^{2,3}$, NOBUKAZU FUJIMOTO ${ }^{4}$, SHIN TANAKA ${ }^{1}$, KEI NAMBA ${ }^{1}$, \\ HIDEJIRO TORIGOE ${ }^{1}$, KAZUHIKO SHIEN ${ }^{1}$, HIROMASA YAMAMOTO ${ }^{1}$, SHUTA TOMIDA ${ }^{5}$, HIROYUKI TAO ${ }^{3,6}$, \\ KAZUNORI OKABE ${ }^{3,6}$, TAKUMI KISHIMOTO ${ }^{4}$ and SHINICHI TOYOOKA ${ }^{1}$
}

\begin{abstract}
${ }^{1}$ Department of General Thoracic Surgery, Okayama University Graduate School of Medicine, Dentistry and Pharmaceutical Sciences, Okayama 700-8558; Departments of ${ }^{2}$ Medical Oncology, and ${ }^{3}$ Clinical Research, National Hospital Organization, Yamaguchi-Ube Medical Center, Ube, Yamaguchi 755-0241;

${ }^{4}$ Department of Respiratory Medicine, Okayama Rosai Hospital, Okayama 702-8055;

${ }^{5}$ Department of Bioinformatics, Okayama University Graduate School of Medicine, Dentistry and Pharmaceutical Sciences, Okayama 700-8558; ${ }^{6}$ Department of Thoracic Surgery, National Hospital Organization, Yamaguchi-Ube Medical Center, Ube, Yamaguchi 755-0241, Japan
\end{abstract}

Received October 18, 2018; Accepted March 8, 2019

DOI: 10.3892/ijo.2019.4768

\begin{abstract}
Malignant pleural mesothelioma (MPM) is a rare malignancy arising from the pleura that is difficult to diagnose, contributing to its dismal prognosis. Previously, we reported that the degree of microRNA (miR)-34b/c methylation in circulating DNA is associated with the development of MPM. Herein, we present a newly developed droplet digital PCR (ddPCR)-based assay for the detection of miR-34b/c methylation in circulating DNA in patients with MPM. We originally prepared two probes within a short amplicon of $60 \mathrm{bp}$, designing one from the positive strand and the other from the complementary strand. The two probes functioned cooperatively, and our established assay detected DNA methylation accurately in the preliminary validation. We subsequently verified this assay using clinical samples. Serum samples from 35 cases of MPM, 29 cases of pleural plaque and 10 healthy volunteers were collected from 3 different
\end{abstract}

Correspondence to: Dr Junichi Soh, Department of General Thoracic Surgery, Okayama University Graduate School of Medicine, Dentistry and Pharmaceutical Sciences, 2-5-1 Shikata-cho, Kita-ku, Okayama 700-8558, Japan

E-mail: soh-j@cc.okayama-u.ac.jp

Abbreviations: MPM, malignant pleural mesothelioma; cfRNA, circulating cell-free RNA; cfDNA, circulating cell-free DNA; ctDNA, circulating cell-free tumor DNA; miR or miRNA, microRNA; ddPCR, droplet digital PCR; PP, pleural plaque; HV, healthy volunteers; LNA, locked nucleic acids; SNPs, single nucleotide polymorphisms

Key words: malignant pleural mesothelioma, droplet digital PCR, microRNA-34b/c, cfDNA, methylation institutions and used in this study. We divided the samples into 2 groups (group $\mathrm{A}, \mathrm{n}=33$; group $\mathrm{B}, \mathrm{n}=41$ ). A receiver-operating characteristic curve analysis using the samples in group A determined the optimal cut-off value for the diagnosis of MPM, with a sensitivity of $76.9 \%$ and a specificity of $90 \%$. On the other hand, the use of the same criterion yielded a sensitivity of $59.1 \%$ and a specificity of $100 \%$ in group B, and corresponding values of 65.7 and $94.9 \%$ for the entire cohort, indicating a moderate sensitivity and a high specificity. In addition, when the analysis was focused on stage II or more advanced MPM, the sensitivity improved to $81.8 \%$, suggesting the possibility that the methylated allele frequency in MPM may be associated with the stage of disease progression. On the whole, the findings of this study indicate that miR-34b/c methylation in circulating DNA is a promising biomarker for the prediction of disease progression in patients with MPM.

\section{Introduction}

Malignant pleural mesothelioma (MPM) is a rare and highly aggressive tumor arising from the pleura or other mesothelial surfaces and is most commonly associated with asbestos exposure, which is known as a major risk factor. Although asbestos use is now prohibited in Western countries, the incidence of MPM is not expected to decrease in the near future due to the long incubation period between asbestos exposure and the onset of MPM $(1,2)$. Moreover, asbestos continues to be used in many developing and emerging economies, such as countries in Southeast Asia, suggesting the possibility of future epidemics of MPM. In the majority of cases, MPM is only diagnosed at an advanced disease stage; therefore, the development of a novel diagnostic approach is warranted $(3,4)$.

Recently, the concept of a 'liquid biopsy' for the diagnosis and monitoring of diseases has attracted attention. Several 
studies have suggested that the individual genetic profiles of cancers are highly heterogeneous and that these profiles can even change during the course of the disease, particularly in response to treatment $(5,6)$. At present, the molecular profiles of patients with solid tumors are generally established using surgically resected or biopsy specimens. However, the use of tissue biopsies is limited by their invasiveness, making it difficult to grasp chronological alterations in molecular profiles and potentially missing some genomic changes. A liquid biopsy originally referred to an analysis of the genomic profiles of circulating tumor cells (7), and this method has attracted particular interest among experts in the field of clinical oncology. This definition has now been extended to include various tumor components, such as circulating cell-free RNA (cfRNA), circulating cell-free DNA (cfDNA), circulating cell-free tumor DNA (ctDNA) and exosomes, and this technique enables clinicians to repeatedly and non-invasively explore real-time changes in the genomic profiles of human cancers.

MicroRNAs (miRNAs or miRs) are a group of small noncoding, endogenous, single-stranded RNAs that play an essential role in the regulation of gene expression. A number of studies have reported that the aberrant hypermethylation of $\mathrm{CpG}$ islands in the promoter regions is closely related to the silencing of tumor-suppressive miRs in several types of cancer (8-11). We previously identified that among several miRs, the epigenetic silencing of miR-34b/c by aberrant methylation in the promoter region plays an important role in the tumorigenesis of MPM (12). miR-34s have been discovered to be direct transcriptional targets of $\mathrm{p} 53$, and to constitute a part of the p53 tumor suppressor network regulating cell cycle arrest, apoptosis and senescence $(13,14)$. As regards the application of miRs as biomarkers, Suzuki et al reported that the aberrant methylation of miR-34b/c in biopsy specimens was a predictive marker of metachronous gastric cancer (15). Wu et al also reported that the detection of methylation in the promoter regions of miR-34s using stool DNA was useful as a screening biomarker for colorectal cancer (16). Additionally, we have previously revealed that the degree of miR-34b/c methylation in serum-circulating DNA is associated with the development of MPM (17). Although the origins of ctDNA differ, these previous studies suggest the possibility that the methylation of the miR-34b/c promoter is a promising biomarker.

In our previous study in 2011, we compared the degree of methylation using MPM cell lines, MPM tissues and nonmalignant mesothelial primary cultures that were established from pleural effusions of cancer-free patients, and we have shown that the promoter of $m i R-34 b / c$ is highly methylated in MPM (12). Based on these findings, the aim of the present study was to apply the miR-34b/c methylation specifically observed in MPM to the diagnosis and prediction of the disease progression. For this purpose, in this study, we established a novel assay with which to detect DNA methylation in the blood using droplet digital PCR (ddPCR) technology, enabling the highly sensitive and quantitative detection of target genes (18). In ddPCR, the input DNA is distributed among approximately 20,000 droplets, and each droplet contains 1 or fewer copies of the target or background DNA; this makes it possible to detect $0.001 \%$ of the target gene from the background DNA (19-21). Our established assay was then verified using serum samples from patients with MPM, pleural plaque patients and healthy volunteers.

\section{Materials and methods}

Clinical samples and cell lines. We collected $>1 \mathrm{ml}$ peripheral blood sample from 35 cases of MPM, 29 cases of pleural plaque (PP) and 10 healthy volunteers (HV) at the Okayama University Hospital (Okayama, Japan), Okayama Rosai Hospital (Okayama, Japan), or the National Hospital Organization, Yamaguchi Ube Medical Center (Yamaguchi, Japan), between January, 2005 and January, 2015. The details are described in Table I. The age of all the healthy volunteers was $>20$ years and healthy individuals who were not any current medications were recruited. None of the participants had a medical history of cancer other than MPM, and all the blood samples were collected before any type of treatment. The blood samples were immediately centrifuged at $5,000 \mathrm{xg}$ for $5 \mathrm{~min}$, and the separated serum samples were stored at $-80^{\circ} \mathrm{C}$ at the respective institutions. In addition, 3 surgically resected MPM specimens obtained from the National Hospital Organization, Yamaguchi Ube Medical Center were also subjected to the methylation assay. All the tissues were frozen in liquid nitrogen immediately after surgery and stored at $-80^{\circ} \mathrm{C}$. This study was conducted with the approval of the Institutional Review Board/Ethical Committee of Okayama University; each of the participants provided written informed consent for the sample collection. All the experiments were performed in accordance with the Declaration of Helsinki.

We also used two human MPM cell lines [NCI-H28 (H28), NCI-H2052 (H2052)] and one human normal mesothelial cell line (MeT-5A) as positive and negative controls, respectively. The H28 and H2052 cells were obtained as kind gifts from Dr Adi F. Gazdar (Hamon Center for Therapeutic Oncology Research and Department of Pathology, University of Texas Southwestern Medical Center at Dallas, Dallas, TX, USA). The MeT-5A cell line was purchased from the American Type Culture Collection (ATCC, Manassas, VA, USA). For the cell lines that had been stored long-term in liquid nitrogen, a DNA fingerprinting analysis by short tandem repeat profiling (the PowerPlex 1.2 System, Promega, Madison, WI, USA) was performed for cell authentication. The cells were maintained in RPMI-1640 medium (Sigma Chemical Co., St. Louis, MO, USA) supplemented with $10 \%$ FBS and cultured in a humidified incubator under $5 \% \mathrm{CO}_{2}$ at $37^{\circ} \mathrm{C}$, and the samples were routinely tested for mycoplasma using the Venor GeM OneStep kit (Minerva Biolabs, Berlin, Germany).

DNA extraction, bisulfite conversion, and bisulfite DNA sequencing. We extracted DNA from the serum samples using the QIAamp Circulating Nucleic Acid kit (Qiagen, Venlo, The Netherlands). The DNA concentrations were quantified using the Qubit 2.0 Fluorometer and Qubit dsDNA HS or BR assay kit (Thermo Fisher Scientific, Waltham, MA, USA). DNA was also extracted from the MPM tissues using the phenol-chloroform method. DNA was extracted from the cell lines using the DNeasy Blood and Tissue kit (Qiagen). Genomic DNA was subjected to bisulfite conversion using the Epitect Bisulfite kit (Qiagen), and the methylation status of miR-34b/c 
Table I. Patient characteristics.

A, Patients in group A

\begin{tabular}{lcc}
\hline Characteristic & MPM $(\mathrm{n}=13)$ & $\mathrm{PP}(\mathrm{n}=20)$ \\
\hline Median (range), years & $71(51-90)$ & $69.5(65-72)$ \\
Sex, male/female & $9 / 4$ & $20 / 0$ \\
Smoking history & & \\
Never & 3 & 5 \\
Former/current & 10 & 15 \\
Histological subtypes & & \\
Epithelioid & 6 & N/A \\
Biphasic & 4 & N/A \\
Sarcomatoid & 3 & N/A \\
Clinical stage & & \\
I & 3 & N/A \\
II & 2 & N/A \\
III & 5 & N/A \\
IV & 1 & N/A \\
Unknown & 2 & N/A \\
& & \\
\hline
\end{tabular}

B, Patients in group B

\begin{tabular}{lccc}
\hline Characteristic & MPM $(\mathrm{n}=22)$ & $\mathrm{PP}(\mathrm{n}=9)$ & $\mathrm{HV}(\mathrm{n}=10)$ \\
\hline Median (range), years & $61.5(49-86)$ & $77(60-86)$ & $31(25-37)$ \\
Sex, male/female & $19 / 3$ & $9 / 0$ & $10 / 0$ \\
Smoking history & & & \\
Never & 6 & 3 & 8 \\
Former/current & 16 & 6 & 2 \\
Histological subtypes & & & \\
Epithelioid & 15 & N/A & N/A \\
Biphasic & 5 & N/A & N/A \\
Sarcomatoid & 2 & N/A & N/A \\
Clinical stage & & & \\
I & 8 & N/A & N/A \\
II & 5 & N/A & N/A \\
III & 5 & N/A & N/A \\
IV & 4 & N/A & N/A \\
\hline
\end{tabular}

MPM, malignant pleural mesothelioma; PP, pleural plaque; HV, healthy volunteer.

was determined using bisulfite DNA sequencing as previously described $(12,17)$. The raw sequence chromatograms were analyzed using Chromas Lite software version 2.6.5 (available at http://technelysium. com.au/wp/ chromas/). The degree of methylation was determined by comparing the intensity of the sequencing electropherogram of cytosine with that of thymine at each of the CpG sites. Based on the electropherograms, we quantified the relative ratios between the heights of each of the waves, as described previously (Fig. 1A) (22), and classified the degree of methylation into three groups, as follows: Low-methylated, degree of methylation $<20 \%$; moderately methylated, degree of methylation between 20 and $70 \%$; and highly methylated, degree of methylation $>70 \%$.

Primers and TaqMan probes. The sequences of the primers and TaqMan probes used in this study were designed based on the nucleotide sequence submitted to GenBank (GenBank accession numbers NR 029839.1 for miR-34b and NR 035765.1 for miR-34c). The melting temperature (Tm) of each primer was calculated using the Oligo Calculator (http://mbcf149.dfci.harvard.edu/docs/oligocalc.html). The primers were synthesized by Invitrogen (Thermo Fisher Scientific, Yokohama, Japan). The primers, including the mixed-base and TaqMan probes containing the locked nucleic acids (LNAs) were designed using the IDT Biophysics software (https://www.idtdna.com/ pages/tools) and were synthesized by Integra ted DNA Technologies KK (Tokyo, Japan).

ddPCR assay for miR-34b/c methylation detection. ddPCR was performed using the QX200 Droplet Digital PCR system (Bio-Rad, Hercules, CA, USA). EpiTect Control DNAs (methylated or unmethylated and bisulfite-converted human DNA, QIAGEN) were used for the assay validation. The total volume of the PCR mixture used for the assay was $22 \mu \mathrm{l}$, containing $10 \mu \mathrm{l}$ of ddPCR Supermix for Probes (No dUTP) (Bio-Rad), $1 \mu \mathrm{M}$ of each primer, $0.25 \mu \mathrm{M}$ of each probe and $200 \mu \mathrm{M}$ of dNTP. As for the amount of DNA, $10 \mu \mathrm{l}$ of cfDNA extracted from the serum was used, while a total of $5 \mathrm{ng}$ of DNA (methylated and bisulfite-converted human control DNA) was applied for the validation of the assay. The PCR conditions were described in detail in our previous study (23). The annealing temperatures were optimized by gradient PCR. The PCR products were then subjected to analysis with the QX-200 droplet reader and QuantaSoft analysis software (Version 1.7.4.0917) (Bio-Rad). The former measures the fluorescence value of each droplet, and the latter measures the number of positive and negative droplets in each sample and calculates the fraction of positive droplets by a Poisson algorithm. QuantaSoft analysis software cannot display the fluorescence intensity of each droplet and standard deviation.

Statistical analysis. All in vitro experiments were performed at least 3 times. Data are represented as the means \pm standard deviation. The concentrations of the target alleles were calculated using QuantaSoft software (Bio-Rad) based on Poisson's distribution. The receiver-operating characteristic (ROC) curve analysis was performed using $\mathrm{JMP}^{\circledR}$ 9.0.0 for Windows (SAS Institute, Inc., Cary, NC, USA). A one-way ANOVA followed by Bonferroni's multiple comparisons test was conducted using GraphPad Prism, version 7 (GraphPad Software, San Diego, CA, USA). Probability values (P-values) $<0.05$ was considered to indicate statistically significant differences.

\section{Results}

Appropriate sequences for primer design. First, we examined candidate sequences suitable for the primer and TaqMan probe design based on some key points, as follows: i) Multiple CpG sites were included in the target sequence to increase the sensitivity; ii) $\mathrm{CpG}$ sites were not included in the primer sequences; and iii) the frequency of single nucleotide polymorphisms (SNPs) 
A
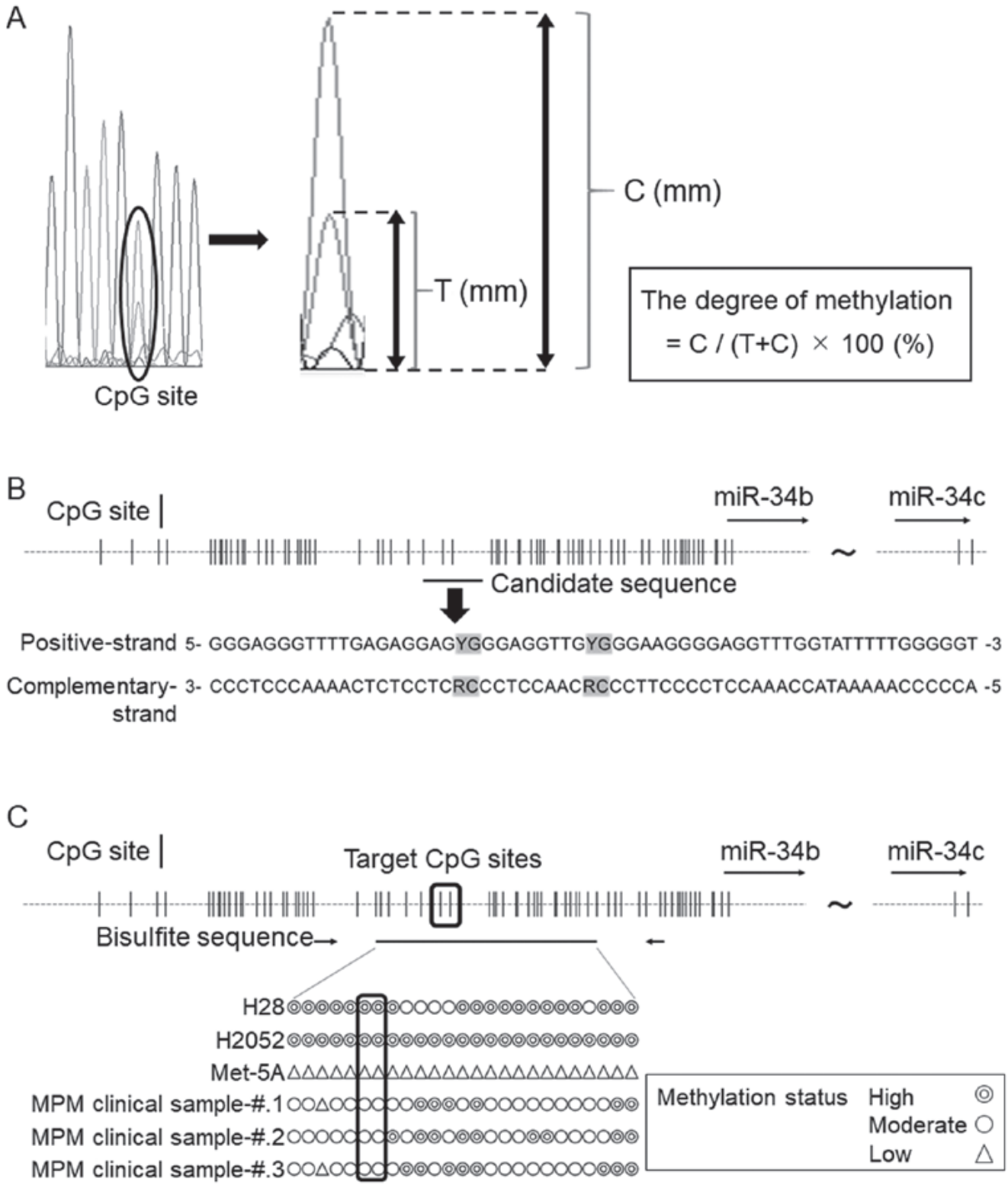

Figure 1. Optimal probe and primer design. (A) Method for calculating the degree of methylation from sequencing electropherograms. C, cytosine (methylated allele); T, thymine (unmethylated allele). (B) Schema of the miR-34b/c promoter region. CpG sites included in the selected sequence are highlighted in gray. G, guanine; A, adenine; T, thymine; C, cytosine; Y, pyrimidine; R, purine. (C) Methylation statuses of 2 MPM cell lines, 1 normal mesothelial cell line, and 3 MPM tissue specimens. Double circles represents a highly methylated status, a single circle represents a moderately methylated status, and a triangle represents a low methylation status. The target $\mathrm{CpG}$ sites and the methylation status are surrounded by the black line. MPM, malignant pleural mesothelioma.

was relatively low in the target sequences. In addition, we made the amplicon size as small as possible to increase the sensitivity of ctDNA detection, as described previously (23). One of the candidate sequences is shown in Fig. 1B, and the SNPs in this target region, as provided by the NCBI dbSNP database (https://www.ncbi.nlm.nih.gov/projects/SNP/), are listed in supplementary Table SI. The possible frequency of SNPs in this region was $\leq 0.02 \%$, which reinforced the validity of this sequence. To confirm the methylation status of the two CpG sites included in this sequence, we performed bisulfite DNA sequencing. The results revealed that both $\mathrm{CpG}$ sites were moderately or highly methylated in both the MPM cell lines and the MPM clinical specimens, but not in the normal mesothelial cell line (Fig. 1C). Based on these results, we designed the primers as shown in Table II. Validation of the primer sets was performed to identify possible non-specific reactions, and we confirmed the specificity of the primers (data not shown).

Probe design and assay validation. Herein, we present a schema representing the principle on which our methylation detection assay was based (Fig. 2). The two CpG sites were detected separately by two TaqMan probes with the same fluorescent dye, and thus we examined the optimal probe design. As both CpG sites in this sequence were located close to each other, we designed one probe based on the sequence of the positive strand (Probe-P), and the other based on the sequence of the complementary strand (Probe-C). In addition, in order to obtain a sufficient match-mismatch Tm difference, the probes were fabricated using LNAs. Based on these concepts, we designed 
Table II. Sequences of primers and probes.

\begin{tabular}{|c|c|c|c|c|c|}
\hline & $\begin{array}{l}\text { Oligo } \\
\text { name }\end{array}$ & Oligo sequences $5^{\prime}$ to $3^{\prime}$ & $\begin{array}{l}\mathrm{Tm} \\
\left({ }^{\circ} \mathrm{C}\right)\end{array}$ & $\begin{array}{l}\text { Product } \\
\text { size (bp) }\end{array}$ & $\begin{array}{l}\text { Match-mismatch } \\
\text { Tm difference }\left({ }^{\circ} \mathrm{C}\right)\end{array}$ \\
\hline \multirow[t]{4}{*}{ Primers } & MPM-Fw & GGGAGGGTTTTGAGAGGAG & 62.54 & 60 & NA \\
\hline & MPM-Rv & AСССССАААААТАССАААСС & 63.28 & & NA \\
\hline & MSP-Fw & AGAGAGTTAGTTTTAGGGTTTGGG & 61.5 & 358 & NA \\
\hline & MSP-Rv & CCTCRAACCCCATTTCAC & 62.95 & & NA \\
\hline \multirow[t]{5}{*}{ Probes } & Probe-P & $\mathrm{FAM} / \mathrm{AC}+\mathrm{CT} \mathrm{C}+\mathrm{CC}+\mathrm{GCT} / \mathrm{IABLFQ}$ & 65.41 & NA & 21.03 \\
\hline & Probe-C1 & FAM/TTG+CGGG+AAGGGG/IABLFQ & 64.07 & NA & 14.75 \\
\hline & Probe-C2 & FAM/TG+CGG+G+A+AGG/IABLFQ & 63.23 & NA & 17.83 \\
\hline & Probe-C3 & FAM/AGGTT+G+C+GGGAAG/IABLFQ & 63.56 & NA & 11.85 \\
\hline & Probe-C4 & FAM/TG+CGGGAAGGGGAG/IABLFQ & 64.65 & NA & 13.29 \\
\hline
\end{tabular}

ddPCR, droplet digital PCR; Tm, melting temperature; IABLFQ, Iowa Black FQ ${ }^{\mathrm{TM}}$; NA, not available; locked nucleic acid, +N; R, mixed base (A or $\mathrm{G})$.

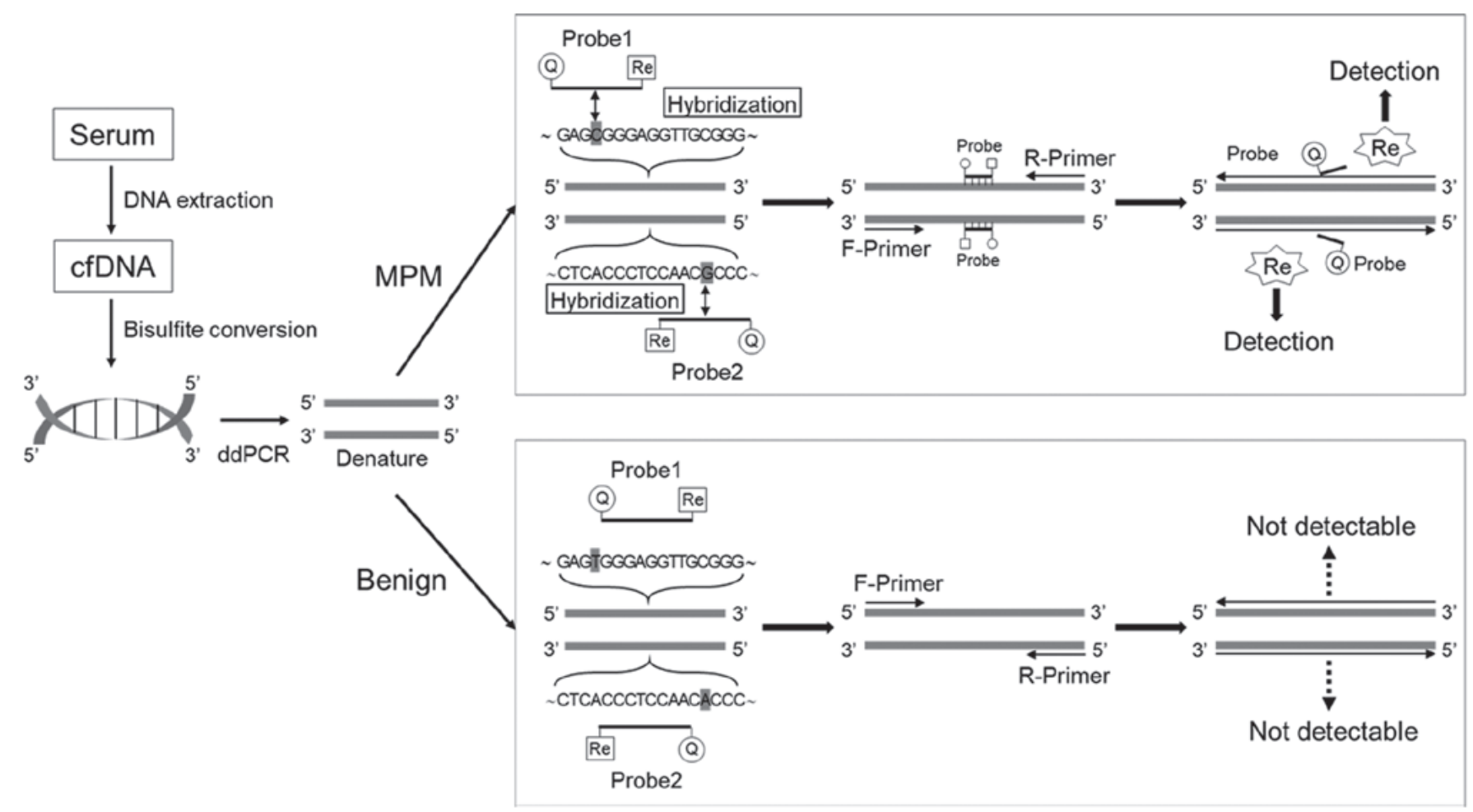

Figure 2. Schematic diagram of the assay established in this study. The target CpG sites of each probe are highlighted in gray. cfDNA, circulating cell-free DNA; MPM, malignant pleural mesothelioma.

several probe sets (Probe-P, Probe-C\#1-4) (Table II). To verify the validity of these probes, and to consider the optimum annealing temperatures, gradient PCR was conducted within the range of $51^{\circ} \mathrm{C}$ to $61^{\circ} \mathrm{C}$. As a result, Probe-P and Probe-C\#1 had a higher fluorescence, compared with the other probes (Fig. 3A and B). Determining whether the two probes would function properly without competition was also important for successful methylation detection. Therefore, to test the interaction between Probe-P and Probe-C\#1, we performed the same experiment using the two probes in combination. We found that the fluorescence intensity was enhanced when the probes were used in combination, suggesting that the probes functioned cooperatively (Fig. 3C and D), and the optimal annealing temperature was determined to be $53.1^{\circ} \mathrm{C}$. Lastly, we confirmed whether this assay could correctly distinguish between methylated and unmethylated DNA. As shown in Fig. 3E and F, the number of droplets with a fluorescence intensity $>3,000$ was noticeably larger in the methylated DNA group.

Clinical application of the established assay. We then evaluated the feasibility of the clinical application of this 

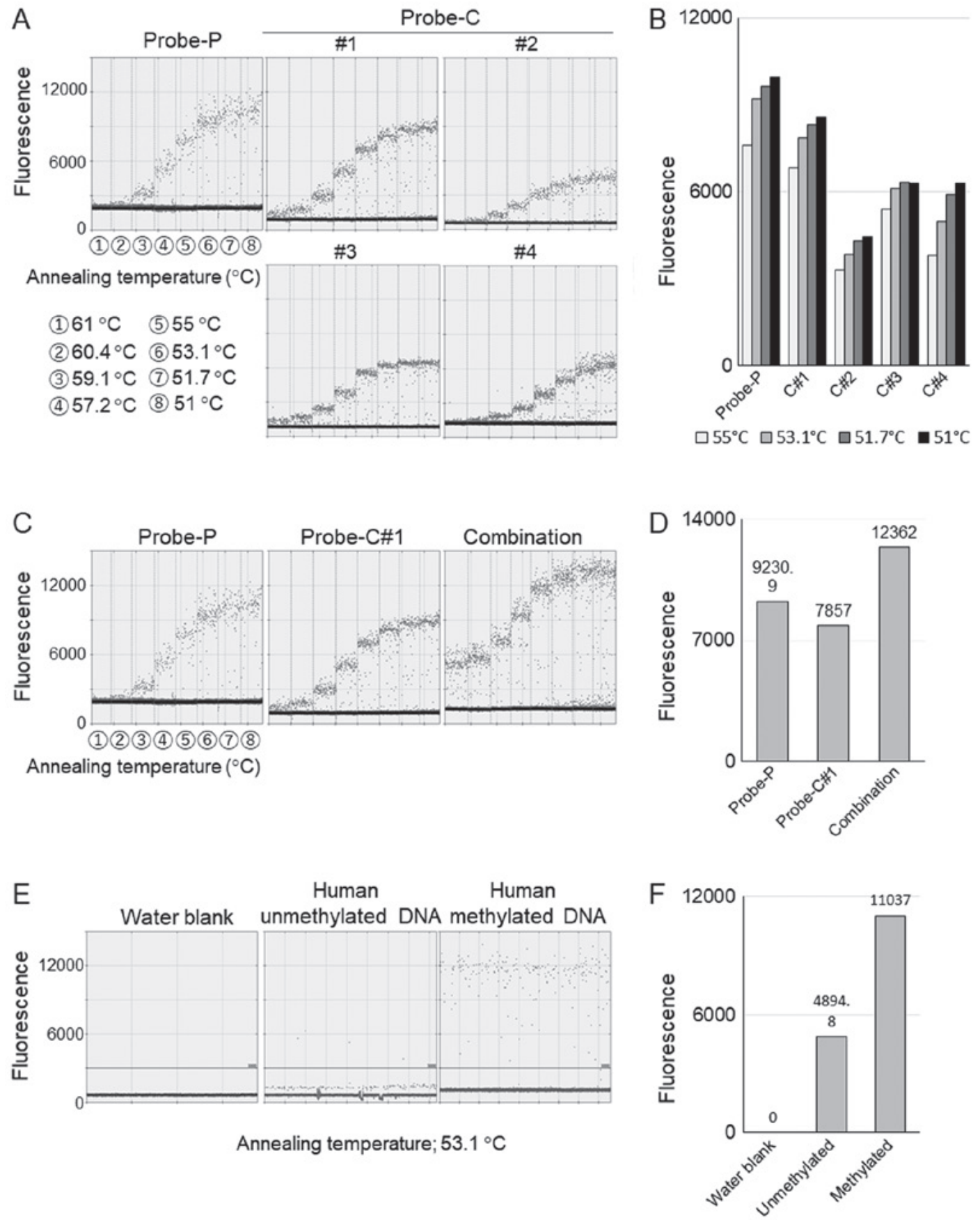

Figure 3. Validation of the established assay. (A) Validation of probes. Gradient PCR was conducted within an annealing temperature range of 51 to $61^{\circ} \mathrm{C}$; the numbered circles indicate the following temperatures: $1,61^{\circ} \mathrm{C} ; 2,60.4^{\circ} \mathrm{C} ; 3,59.1^{\circ} \mathrm{C} ; 4,57.2^{\circ} \mathrm{C} ; 5,55^{\circ} \mathrm{C} ; 6,53.1^{\circ} \mathrm{C} ; 7,51.7^{\circ} \mathrm{C}$; and $8,51^{\circ} \mathrm{C}$. Probe-P represents the probe designed based on the sequence of the positive strand, and Probe-C represents the probe designed based on the sequence of the complementary strand. (B) The mean fluorescence values of droplets with a fluorescence intensity of over 3,000. (C) Verification of combined use of the probes. The annealing temperature was ranged from 51 to $61^{\circ} \mathrm{C}$; the numbered circles indicate the following temperatures: $1,61^{\circ} \mathrm{C} ; 2,60.4^{\circ} \mathrm{C} ; 3,59.1^{\circ} \mathrm{C} ; 4,57.2^{\circ} \mathrm{C} ; 5,55^{\circ} \mathrm{C} ; 6,53.1^{\circ} \mathrm{C}$; $7,51.7^{\circ} \mathrm{C}$; and $8,51^{\circ} \mathrm{C}$. The use of Probe-P and Probe-C\#1 in combination was associated with an enhanced fluorescence intensity, compared with that of each probe alone (right panel). (D) The mean fluorescence values of droplets with a fluorescence intensity of over 3,000 at annealing temperature of $53.1^{\circ} \mathrm{C}$. (E) Verification of established assay using methylated and non-methylated human DNA. (F) The mean fluorescence values of droplets with a fluorescence intensity of over 3,000 at annealing temperature of $53.1^{\circ} \mathrm{C}$.

assay. We divided the serum samples (35 cases of MPM, 29 cases of PP and $10 \mathrm{HVs})$ into group A ( $\mathrm{n}=33)$ and group B $(\mathrm{n}=41)$ according to their collection site: Samples obtained from the Okayama Rosai Hospital were classified as group A, while those obtained from the other two institutions were classified as group B. The characteristics of the patients in the 2 groups are summarized in Table I. The median concentration of cfDNA extracted from the serum was
$1.47 \mathrm{ng} / \mu 1$ for the MPM cases and $1.44 \mathrm{ng} / \mu 1$ for the others. Firstly, to determine the positive criterion, we conducted an ROC curve analysis comparing the MPM cases with other non-malignant cases using samples from group A (Fig. 4A and Table SII). The results indicated that the presence of at least 3 droplets with a fluorescence of over a threshold value of 7,000 was the optimal cut-off for the diagnosis of MPM, with a sensitivity of $76.9 \%$ and a specificity of $90 \%$. 

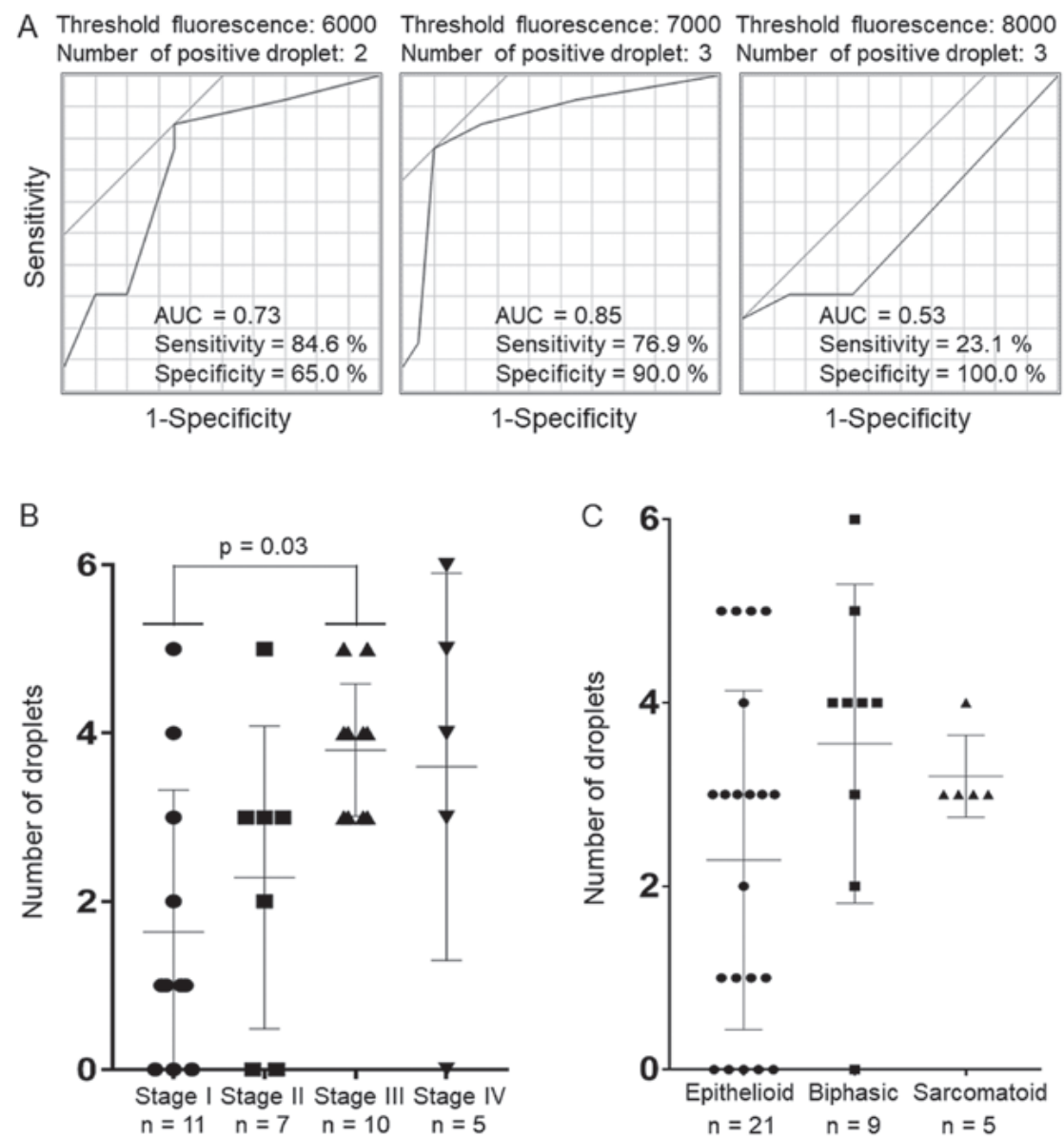

Figure 4. Validation for clinical application of the established assay. (A) Comparison of receiver-operating characteristic (ROC) curves among threshold fluorescence values of 6,000-8,000. ROC curves for the positive criterion of methylation detection are shown. AUC, area under the ROC curve. (B) Number of droplets with fluorescence over the threshold value of 7,000 according to the clinical stage of MPM. The bars represent the mean with standard deviation. MPM, malignant pleural mesothelioma. (C) Number of droplets with fluorescence over the threshold value of 7,000 according to the histological subtypes of MPM. The bars represent the means \pm standard deviation.

Subsequently, we evaluated the validity of this criterion. The results are shown in Table III. The sensitivity and specificity for the diagnosis of MPM in the group B samples were 59.1 and $100 \%$, respectively, while those for the entire cohort were 65.7 and $94.9 \%$, respectively, indicating a moderate sensitivity and a high specificity. In addition, when we focused on the diagnosis of only stage II or more advanced MPM, the sensitivity increased to $81.8 \%$. Actually, there were significant differences in the number of droplets with fluorescence values of at least 7,000 per case among stages (one-way ANOVA, $\mathrm{P}=0.02$ ) (Bonferroni's post hoc test; stage I vs. stage II, $\mathrm{P}>0.99$; stage I vs. stage III, $\mathrm{P}=0.03$; stage I vs. stage IV, $\mathrm{P}>0.18$; stage II vs. stage III, $\mathrm{P}=0.39$; stage II vs. stage IV, $\mathrm{P}>0.99$; stage III vs. stage IV, $\mathrm{P}>0.99$ ), suggesting that the methylated allele frequency may be associated with the stage of MPM progression (Fig. 4B). On the other hand, methylation was not detected in one case with clinical stage IV MPM. We also assessed whether the histological subtypes were associated with the methylated allele frequency. However, no significant difference in the methylated allele frequency was observed among the histological subtypes (one-way ANOVA, $\mathrm{P}=0.16$ ) (Bonferroni's post-hoc test; epithelioid vs. biphasic, $\mathrm{P}=0.21$; epithelioid vs. sarcomatoid, $\mathrm{P}=0.87$; biphasic vs. sarcomatoid, $\mathrm{P}>0.99$ ) (Fig. 4C).
Table III. Assay sensitivity and specificity of each group.

\section{MPM PP or HV}

Group A ( $n=33)$

Positive

Negative

3 18

Sensitivity, $76.9 \%$; specificity, $90.0 \%$

Group B ( $\mathrm{n}=41)$

Positive

Negative

Sensitivity, $59.1 \%$; specificity, $100 \%$

Entire cohort $(\mathrm{n}=74)$

Positive

Negative

Sensitivity, $65.7 \%$; specificity, $94.9 \%$

Stage II or more advanced MPM ( $\mathrm{n}=61)$

Positive

Negative

4 37

Sensitivity, $81.8 \%$; specificity, $94.9 \%$

MPM, malignant pleural mesothelioma; PP, pleural plaque; HV, healthy volunteer. 


\section{Discussion}

In this study, we established a TaqMan-based ddPCR assay for the detection of the methylation of the miR-34b/c promoter region in circulating DNA. The design of the two probes, one from the positive strand and the other from the complementary strand, allowed the successful detection of the methylation of the two $\mathrm{CpG}$ sites located close to each other, with an overall specificity of $94.9 \%$. Although the sensitivity of our assay was limited to $65.7 \%$, when the analysis was focused on the detection of stage II or more advanced cases of MPM, the sensitivity increased to $81.8 \%$, and there was a tendency that the methylated allele frequency was higher in more advanced MPM. These findings suggest that the methylation status may be positively associated with the stage of MPM progression and that it may be useful for predicting tumor progression. As for the association between the methylation status of tumor suppressor genes and the disease progression, Jezkova et al also reported that the hypermethylation of RASSF1A and PITX2, which are known for the tumor suppressor gene in breast cancer, is significantly associated with tumor stage in breast cancer patients (24). On the other hand, Guo et al mentioned that there was no significant difference in the methylation status of HOXD10, which functions as a tumor suppressor in hepatocellular carcinoma (HCC), between the HCC patients with stage I and II and those with stage III and stage IV (25). Thus, whether the degree of the promoter methylation can predict the tumor progression may depend on the type of cancer and gene. In addition, in our series, methylation was not detected in one case despite the patient having clinical stage IV MPM; therefore, further studies of the tumor characteristics that may be particularly related to the degree of methylation are required.

It is well known that both chemotherapy and radiotherapy induce DNA methylation changes, and chemotherapy or radiation-induced alterations in DNA methylation result in changes in the biological response to the treatment. Recently, Flanagan et al reported that platinum-based chemotherapy induces DNA methylation changes in blood DNA, and the methylation levels in blood DNA at the time of relapse can reflect the clinical outcome of cancer patients (26). Sun et al also reported that the promoter methylation level of RASSF1A was affected by oxaliplatin-based chemotherapy, and the methylation status in blood DNA can be used to predict the outcome of patients with colorectal cancer (27). Thus, the influences of treatments on methylation statuses are a very important issue that should be examined in the future, and $\mathrm{miR}-34 \mathrm{~b} / \mathrm{c}$ is no exception. Therefore, the samples that were used in the present study were collected before any treatment was administered.

Several circulating biomarkers have been reported for the diagnosis of MPM, including the soluble mesothelin-related peptides, osteopontin, fibulin-3 and miRs $(28,29)$. As for protein markers, while they exhibit excellent specificity, their poor sensitivity reduces their diagnostic usefulness (30-32). As regards circulating miRs, although some miRs exhibit diagnostic potential for MPM, there are problems, such as their origin (whether they are derived from tumor cells or hematopoietic cells is still controversial) that need to be resolved, and the majority of the analyses of cfRNA in the blood remain exploratory (21). On the other hand, few studies have reported the usefulness of a diagnostic method targeting the degree of methylation of DNA, not the miR or protein itself, for MPM. Several studies have reported the existence of a strong association between the methylation status in tumor tissue samples and that in ctDNA from blood samples; therefore, targeting ctDNA methylation is reasonable (33-36). As suggested by previous studies, a combination of various approaches could be useful to increase the sensitivity, and targeting circulating methylated DNA may be a worthwhile addition $(28,29)$.

Whereas we used a SYBR-Green-based real-time MSP assay ( 48 wells/sample) in our previous study, we adopted a TaqMan-based ddPCR assay (20,000 droplets/well) in the present study to improve the specificity and accuracy of the detection of methylated DNA from amongst a large amount of background DNA. As a result, the specificity of the assay was improved to $94.9 \%$, compared with that in our previous study. On the other hand, the sensitivity of the established assay was limited to $65.7 \%$. The median dosage of cfDNA in this study was approximately $15 \mathrm{ng} / \mathrm{sample}$, corresponding to 4,500 haploid genome equivalents. Considering the capability of ddPCR, it is possible to process larger amounts of cfDNA. Increasing the dosage of DNA may lead to an improvement in sensitivity. Recently, cfDNA in body fluids other than blood, such as urine or stool, has also attracted attention as useful biomarkers of cancer $(37,38)$. The collection of these samples offers the advantage of being truly non-invasive and allowing large sample volumes to be collected, which may compensate for the disadvantage of the rather limited amount of cfDNA in the blood. In addition, the concentration of ctDNA is one of the key factors for successful cancer detection using a liquid biopsy, and it is well known that the proportion of ctDNA in cfDNA varies among patients depending on the tumor localization, size, vascularization, and clearance, ranging from $<0.005$ to $90 \%$ in several types of cancer (39-42). However, the association between ctDNA and total cfDNA in MPM remains unclear; therefore, further investigation of this issue using liquid biopsies in patients with MPM will be our next task.

This study had some limitations. First, the sample size was too small to enable a definitive conclusion, and the groups in this study were not matched for background characteristics, such as age and sex. Considering the rarity of MPM, large clinical trials would be preferable. Second, plasma samples are more suitable than serum samples for cfDNA analyses due to the lower background level of wild-type DNA in the former $(21,43)$. Therefore, our established assay should be validated using plasma samples. These factors could have introduced some bias to our results.

In conclusion, in this study, we established a novel detection system for the promoter methylation of $\mathrm{miR}-34 \mathrm{~b} / \mathrm{c}$ using ddPCR. Our findings suggest the possibility that miR-34b/c methylation in ctDNA could be a promising circulating biomarker for the prediction of disease progression in MPM.

\section{Acknowledgements}

The authors would like to thank Dr Takehiro Matsubara (Biobank, Okayama University Graduate School of Medicine, Dentistry and Pharmaceutical Sciences, Okayama, Japan), 
Ms. Yoko Kojima (Research Center for Asbestos-related Disease, Okayama Rosai Hospital), and Ms. Fumiko Isobe (Department of General Thoracic Surgery and Breast and Endocrinological Surgery, Okayama University Graduate School of Medicine, Dentistry and Pharmaceutical Sciences, Okayama, Japan) for their technical support.

\section{Funding}

This study was supported by a Management Expenses Grants.

\section{Availability of data and materials}

The datasets analyzed during the current study are available from the corresponding author on reasonable request.

\section{Authors' contributions}

HS, JS, HY, KS and SToyooka conceived and designed experiments. HS, STanaka, HTo and KN conducted the experiments. HS and STomida analyzed data and prepared the figures. KA, NF, HTa, KO and TK contributed to the sample collection. HS, JS and SToyooka wrote the manuscript. All authors discussed the results and commented on the manuscript. All authors have read and approved the final manuscript.

\section{Ethics approval and consent to participate}

This study was conducted with the approval of the Institutional Review Board/Ethical Committee of Okayama University; each of the participants provided written informed consent for the sample collection. All the experiments were performed in accordance with the Declaration of Helsinki.

\section{Patient consent for publication}

Not applicable.

\section{Competing interests}

The authors declare that they have no competing interests.

\section{References}

1. Henley SJ, Larson TC, Wu M, Antao VC, Lewis M, Pinheiro GA and Eheman C: Mesothelioma incidence in 50 states and the District of Columbia, United States, 2003-2008. Int J Occup Environ Health 19: 1-10, 2013.

2. Scherpereel A: Malignant pleural mesothelioma: new treatments, new hopes? Eur Respir J 49: 1700319, 2017.

3. Goudar RK: Review of pemetrexed in combination with cisplatin for the treatment of malignant pleural mesothelioma. Ther Clin Risk Manag 4: 205-211, 2008.

4. Toyooka S, Kishimoto T and Date H: Advances in the molecular biology of malignant mesothelioma. Acta Med Okayama 62: 1-7, 2008.

5. Russo M, Siravegna G, Blaszkowsky LS, Corti G, Crisafulli G, Ahronian LG, Mussolin B, Kwak EL, Buscarino M, Lazzari L, et al: Tumor heterogeneity and lesion-specific response to targeted therapy in colorectal cancer. Cancer Discov 6: 147-153, 2016.

6. Murtaza M,Dawson SJ, Pogrebniak K, Rueda OM, ProvenzanoE, Grant J, Chin SF, Tsui DW, Marass F, Gale D, et al: Multifocal clonal evolution characterized using circulating tumour DNA in a case of metastatic breast cancer. Nat Commun 6: 8760, 2015.
7. Alix-Panabières $\mathrm{C}$, Schwarzenbach $\mathrm{H}$ and Pantel K: Circulating tumor cells and circulating tumor DNA. Annu Rev Med 63: 199-215, 2012.

8. Loginov VI, Pronina IV, Burdennyy AM, Filippova EA, Kazubskaya TP, Kushlinsky DN, Utkin DO, Khodyrev DS, Kushlinskii NE, Dmitriev AA, et al: Novel miRNA genes deregulated by aberrant methylation in ovarian carcinoma are involved in metastasis. Gene 662: 28-36, 2018.

9. Tian Y, Wei W, Li L and Yang R: Down-regulation of miR-148a promotes metastasis by DNA methylation and is associated with prognosis of skin cancer by targeting TGIF2. Med Sci Monit 21: 3798-3805, 2015

10. Croce CM: Causes and consequences of microRNA dysregulation in cancer. Nat Rev Genet 10: 704-714, 2009.

11. Toyota M, Suzuki H, Sasaki Y, Maruyama R, Imai K, Shinomura Y and Tokino T: Epigenetic silencing of microRNA-34b/c and $\mathrm{B}$-cell translocation gene 4 is associated with $\mathrm{CpG}$ island methylation in colorectal cancer. Cancer Res 68: 4123-4132, 2008.

12. Kubo T, Toyooka S, Tsukuda K, Sakaguchi M, Fukazawa T, Soh J, Asano H, Ueno T, Muraoka T, Yamamoto $\mathrm{H}$, et al: Epigenetic silencing of microRNA-34b/c plays an important role in the pathogenesis of malignant pleural mesothelioma. Clin Cancer Res 17: 4965-4974, 2011.

13. He L, He X, Lim LP, de Stanchina E, Xuan Z, Liang Y, Xue W, Zender L, Magnus J, Ridzon D, et al: A microRNA component of the p53 tumour suppressor network. Nature 447: 1130-1134, 2007.

14. Corney DC, Flesken-Nikitin A, Godwin AK, Wang W and Nikitin AY: MicroRNA-34b and microRNA-34c are targets of p53 and cooperate in control of cell proliferation and adhesion-independent growth. Cancer Res 67: 8433-8438, 2007.

15. Suzuki R, Yamamoto E, Nojima M, Maruyama R, Yamano HO, Yoshikawa K, Kimura T, Harada T, Ashida M, Niinuma T, et al: Aberrant methylation of microRNA-34b/c is a predictive marker of metachronous gastric cancer risk. J Gastroenterol 49: 1135-1144, 2014.

16. Wu XD, Song YC, Cao PL, Zhang H, Guo Q, Yan R, Diao DM, Cheng Y and Dang CX: Detection of miR-34a and miR-34b/c in stool sample as potential screening biomarkers for noninvasive diagnosis of colorectal cancer. Med Oncol 31: 894, 2014.

17. Muraoka T, Soh J, Toyooka S, Aoe K, Fujimoto N, Hashida S, Maki Y, Tanaka N, Shien K, Furukawa M, et al: The degree of microRNA-34b/c methylation in serum-circulating DNA is associated with malignant pleural mesothelioma. Lung Cancer 82: 485-490, 2013.

18. Oxnard GR, Paweletz CP, Kuang Y, Mach SL, O'Connell A, Messineo MM, Luke JJ, Butaney M, Kirschmeier P, Jackman DM, et al: Noninvasive detection of response and resistance in EGFR-mutant lung cancer using quantitative next-generation genotyping of cell-free plasma DNA. Clin Cancer Res 20: 1698-1705, 2014.

19. Hindson BJ, Ness KD, Masquelier DA, Belgrader P, Heredia NJ, Makarewicz AJ, Bright IJ, Lucero MY, Hiddessen AL, Legler TC, et al: High-throughput droplet digital PCR system for absolute quantitation of DNA copy number. Anal Chem 83: 8604-8610, 2011.

20. Sanmamed MF, Fernández-Landázuri S, Rodríguez C, Zárate R, Lozano MD, Zubiri L, Perez-Gracia JL, Martín-Algarra S and González A: Quantitative cell-free circulating BRAFV600E mutation analysis by use of droplet digital PCR in the follow-up of patients with melanoma being treated with BRAF inhibitors. Clin Chem 61: 297-304, 2015.

21. Siravegna G, Marsoni S, Siena S and Bardelli A: Integrating liquid biopsies into the management of cancer. Nat Rev Clin Oncol 14: 531-548, 2017

22. Soh J, Okumura N, Lockwood WW, Yamamoto H, Shigematsu H, Zhang W, Chari R, Shames DS, Tang X, MacAulay C, et al: Oncogene mutations, copy number gains and mutant allele specific imbalance (MASI) frequently occur together in tumor cells. PLoS One 4: e7464, 2009.

23. Suzawa K, Yamamoto H, Ohashi K, Hashida S, Tomida S, Kubo T, Maki Y, Soh J, Tsukuda K, Kiura K, et al: Optimal method for quantitative detection of plasma EGFR T790M mutation using droplet digital PCR system. Oncol Rep 37: 3100-3106, 2017

24. Jezkova E, Kajo K, Zubor P, Grendar M, Malicherova B, Mendelova A, Dokus K, Lasabova Z, Plank L and Danko J: Methylation in promoter regions of PITX2 and RASSF1A genes in association with clinicopathological features in breast cancer patients. Tumour Biol 37: 15707-15718, 2016. 
25. Guo Y, Peng Y, Gao D, Zhang M, Yang W, Linghu E, Herman JG, Fuks F, Dong G and Guo M: Silencing HOXD10 by promoter region hypermethylation activates ERK signaling in hepatocellular carcinoma. Clin Epigenetics 9: 116, 2017.

26. Flanagan JM, Wilson A, Koo C, Masrour N, Gallon J, Loomis E, Flower K, Wilhelm-Benartzi C, Hergovich A, Cunnea P, et al: Platinum-based chemotherapy induces methylation changes in blood DNA associated with overall survival in patients with ovarian cancer. Clin Cancer Res 23: 2213-2222, 2017.

27. Sun X, Yuan W, Hao F and Zhuang W: Promoter methylation of RASSF1A indicates prognosis for patients with stage II and III colorectal cancer treated with oxaliplatin-based chemotherapy. Med Sci Monit 23: 5389-5395, 2017.

28. Cristaudo A, Bonotti A, Guglielmi G, Fallahi P and Foddis R: Serum mesothelin and other biomarkers: What have we learned in the last decade? J Thorac Dis 10 (Suppl 2): S353-S359, 2018.

29. Bruno R, Alì G and Fontanini G: Molecular markers and new diagnostic methods to differentiate malignant from benign mesothelial pleural proliferations: A literature review. J Thorac Dis 10 (Suppl 2): S342-S352, 2018.

30. Hu ZD, Liu XF, Liu XC, Ding CM and Hu CJ: Diagnostic accuracy of osteopontin for malignant pleural mesothelioma: A systematic review and meta-analysis. Clin Chimica Acta 433: 44-48, 2014.

31. Creaney J, Dick IM, Meniawy TM, Leong SL, Leon JS Demelker Y, Segal A, Musk AW, Lee YC, Skates SJ, et al: Comparison of fibulin-3 and mesothelin as markers in malignant mesothelioma. Thorax 69: 895-902, 2014.

32. van Zandwijk N, Clarke C, Henderson D, Musk AW, Fong K, Nowak A, Loneragan R, McCaughan B, Boyer M, Feigen M, et al: Guidelines for the diagnosis and treatment of malignant pleural mesothelioma. J Thorac Dis 5: E254-E307, 2013.

33. Xu RH, Wei W, Krawczyk M, Wang W, Luo H, Flagg K, Yi S, Shi W, Quan Q, Li K, et al: Circulating tumour DNA methylation markers for diagnosis and prognosis of hepatocellular carcinoma. Nat Mater 16: 1155-1161, 2017

34. Pishvaian MJ, Joseph Bender R, Matrisian LM, Rahib L, Hendifar A, Hoos WA, Mikhail S, Chung V, Picozzi V, Heartwell C, et al: A pilot study evaluating concordance between blood-based and patient-matched tumor molecular testing within pancreatic cancer patients participating in the Know Your Tumor (KYT) initiative. Oncotarget 8: 83446-83456, 2016.
35. Liggett T, Melnikov A, Yi QL, Replogle C, Brand R, Kaul K, Talamonti M, Abrams RA and Levenson V: Differential methylation of cell-free circulating DNA among patients with pancreatic cancer versus chronic pancreatitis. Cancer 116: 1674-1680, 2010

36. Hoque MO, Feng Q, Toure P, Dem A, Critchlow CW, Hawes SE, Wood T, Jeronimo C, Rosenbaum E, Stern J, et al: Detection of aberrant methylation of four genes in plasma DNA for the detection of breast cancer. J Clin Oncol 24: 4262-4269, 2006.

37. Worm Ørntoft MB: Review of blood-based colorectal cancer screening: how far are circulating cell-free DNA methylation markers from clinical implementation? Clin Colorectal Cancer 17: e415-e433, 2018.

38. Stewart CM, Kothari PD, Mouliere F, Mair R, Somnay S, Benayed R, Zehir A, Weigelt B, Dawson SJ, Arcila ME, et al: The value of cell-free DNA for molecular pathology. J Pathol 244: 616-627, 2018.

39. Diehl F, Schmidt K, Choti MA, Romans K, Goodman S, Li M, Thornton K, Agrawal N, Sokoll L, Szabo SA, et al: Circulating mutant DNA to assess tumor dynamics. Nat Med 14: 985-990, 2008.

40. Bettegowda C, Sausen M, Leary RJ, Kinde I, Wang Y, Agrawal N, Bartlett BR, Wang H, Luber B, Alani RM, et al: Detection of circulating tumor DNA in early- and late-stage human malignancies. Sci Transl Med 6: 224ra24, 2014.

41. Thierry AR, Mouliere F, El Messaoudi S, Mollevi C, Lopez-Crapez E, Rolet F, Gillet B, Gongora C, Dechelotte P, Robert B, et al: Clinical validation of the detection of KRAS and BRAF mutations from circulating tumor DNA. Nat Med 20: 430-435, 2014.

42. Zeng H, He B, Yi C and Peng J: Liquid biopsies: DNA methylation analyses in circulating cell-free DNA. J Genet Genomics 45: $185-192,2018$

43. Jung M, Klotzek S, Lewandowski M, Fleischhacker $M$ and Jung K: Changes in concentration of DNA in serum and plasma during storage of blood samples. Clin Chem 49: 1028-1029, 2003. 